\title{
AUTHENTICITY OF PRIMARY CLINICAL DIAGNOSIS PARKINSON'S DISEASE IN SERIES OF 5583 PATIENTS
}

\author{
Deleva N., B. Ivanov, A. Kaprelyan, I. Dimitrov \\ I Clinic of Neurology, Department of Neurology, \\ Prof. Paraskev Stoyanov Medical University of Varna
}

Reviewed by: Prof. A. Klisarova

\begin{abstract}
The recognition of Parkinson's disease (PD) in the initial stages is often difficult, especially when only part of the symptoms is present. The early and accurate diagnosis is important for both therapeutic and economical reasons. Aim of the present study was to establish the authenticity of primary clinical diagnosis PD in patients referred to our clinic during a 5-year period (2004-2008). A total of 5583 patients ( 3120 males and 2463 females), mean age 73.2 \pm 14.6 , and mean duration of disease $8.4 \pm 4.2$ years were included in the study. Neurological examination, EMG, neuroimaging, and biochemical analysis have been performed. PD was confirmed in $3807(68 \%)$ patients, Essential tremor (ET) in $1504(27 \%)$, and other diseases in 276 patients $(5 \%)$. Based on our own results and literature review we suggested that the strictly observance to the diagnostic criteria improves the authenticity of primary clinical diagnosis PD and has an essential medico-social significance. Special attention should be paid to ET, the most frequent reason for hyperdiagnosis of PD.
\end{abstract}

Key words: authenticity, clinical diagnosis, Parkinson's disease, hyperdiagnosis

\section{INTRODUCTION}

Parkinson's disease is one of the most common neurodegenerative diseases with enormous medical, social, and economical significance. The diagnosis is extremely clinical and often is difficult in the initial stages, when only part of the symptoms is present. Differential diagnosis mostly includes Essential tremor (ET), vascular and drug-induced parkinsonism, and Parkinson-plus syndromes (4). Therefore, the early and accurate diagnosis is very important. Furthermore in recent clinical practice more and more medications with potential neuroprotective and delaying disease progression effect are introduced. It is known that to apply the complete set of clinical criteria in the daily outpatient practice is extremely difficult. Accordingly, in our country both national expert commissions and university centers for diagnosis and treatment of PD are founded. In correspondence, we decided to establish the authenticity of primary clinical diagnosis PD in patients referred to our PD consultative center.

\section{MATERIAL AND METHODS}

A total of 5583 patients (3120 males and 2463 females), mean age $73.2 \pm 14.6$ years, and medium duration of dis-

Address for correspondence:

N. Deleva, Dept. of Neurology, Prof. P.Stoyanov Medical university of Varna, 55 Marin Drinov Str.; 9002 Varna, Bulgaria

e-mail: ndeleva@mail.bg ease $8.4 \pm 4.2$ years with primary outpatient diagnosis PD were included in the present study. All individuals were referred to our Center for diagnosis and treatment of Parkinson's disease during a 5-year period (2004-2008). Complete neurological examination, pharmacological test with dispersible tablet of Levodopa, EMG, neuroimaging, and biochemical analysis has been performed. The final diagnosis was in accordance with the criteria of Bulgarian consensus for diagnosis and treatment of PD.

\section{RESULTS}

Diagnosis PD was confirmed in 3807 (68\%) patients (2132 males and 1675 females), mean age $75.4 \pm 12.8$, and disease duration $7.5 \pm 3.7$ years. According to the Hoehn-Yahr (HY) scale 647 (17\%) of patients were in stage I, 1561 $(41 \%)$ in ${ }^{22}, 1142(30 \%)$ in ${ }^{222}$, and $457(12 \%)$ in stage ${ }^{2} \mathrm{~V}$ (Fig. 1). The average points of total UPDRS sum was 21.5 \pm 2.8 .

ET was diagnosed in 1504 (27\%) patients (643 males and 861 females), mean age $67.8 \pm 4.7$ years, and disease duration $9.7 \pm 4.1$ years. The rest diagnoses $(5 \%)$ include vascular parkinsonism $224(4 \%)$ patients, drug-induced parkinsonism $19(0.4 \%)$, Wilson's disease $14(0.2 \%)$, multisystem atrophy $4(0.1 \%)$, chorea $6(0.1 \%)$ and other diseases without parkinsonian syndrome $9(0.2 \%)$ patients (Fig. 2). 


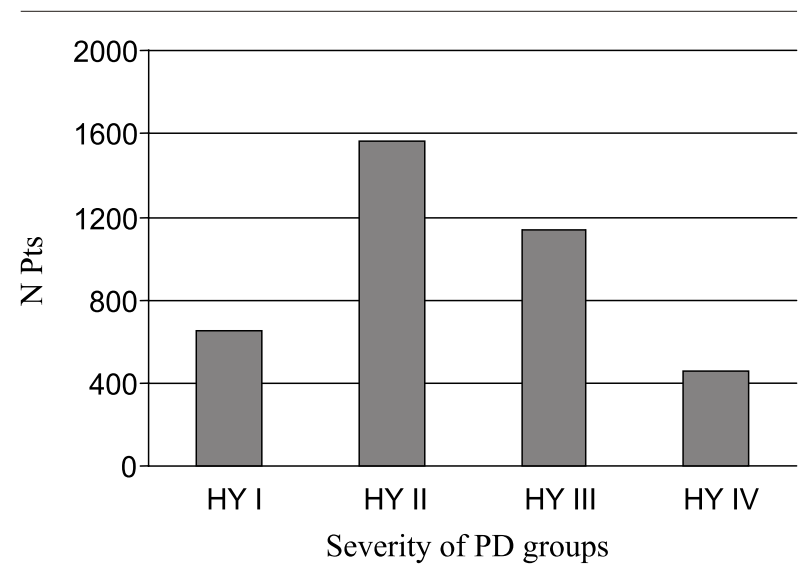

Fig. 1. Distribution of patients according to Hoehn-Yahr scale.

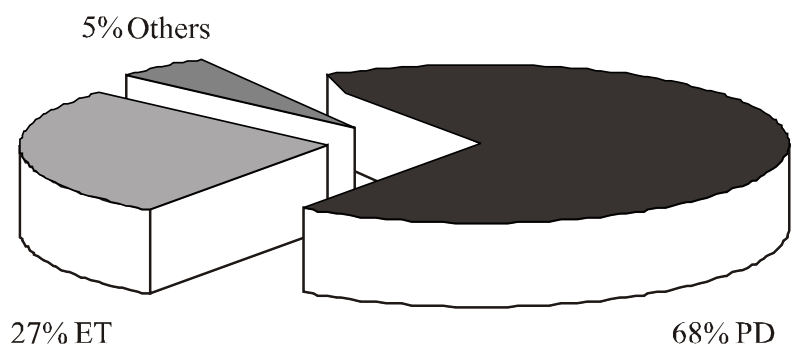

Fig. 2. Relative distribution of established diagnosis.

\section{DISCUSSION}

The analysis of our own results reveals high percentage of wrong primary diagnosis PD. According to the literature the hyperdiagnosis varies between $8-35 \%$ of the cases. The most common misdiagnosis is ET, which is 10 times more often. Our findings are in accordance with these epidemiological data.

Facts exist that these patients usually receive antiparkinonian drugs for a long period that is without justification from the point of lack of efficacy, the potential side-effects, and high medication price $(2,5)$.

In our study we find a high percentage of patients even without clinical symptoms of Parkinson syndrome, while the group of patients with Wilson's disease and Parkinson-plus syndrome is relatively smaller. Our results correspond to previously reported data revealing relatively high percentage of false clinical diagnosis $(1,3)$.

PD diagnosis is based on strongly defined criteria from international consensus guidelines. Although their strictly observance, the $100 \%$ authenticity is not possible. It is considered that the diagnosis established even by experts of movement disorders could be wrong in $8 \%$ of the cases (6).

\section{CONCLUSION}

Based on our own results we suggest that the percentage of false outpatient PD diagnosis is relatively high. Therefore, the strictly observance to the diagnostic criteria of national consensus may improve the authenticity of primary clinical diagnosis. Accordingly, the reduction of patients with PD to their real number has an important medico-social and economical significance.

\section{REFERENCES}

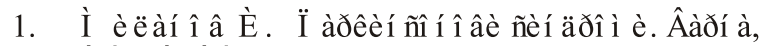
ÈÊ ÑÒÅÍ î , 2005.

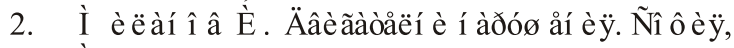
İ åä. è ô èçê., 2007.

3. İ è èài î â È. Äåãåí åđàòêấ è çàái ëÿâài èỹ í à í åđâí àò ñèñoài à. İ àđêèi n̂î́i î âè neéí äđî ì è. Â:

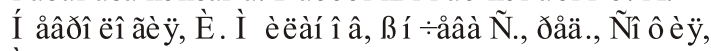
İ åä. è ồèçe., 2007, 495-541.

4. Braak H. Staging of the intracerebral inclusion body pathology associated with idiopathic PD. J Neurol, 2002, 249, suppl 3, 1-5.

5. Braune S., Reinhardt M., Schnitzer R., Riedel A., Lucking C. Cardiac uptake of 123I MIBG separates Parkinson`s disease from multiple system atrophy. Neurology, 1999, 53, 1020-25.

6. Catafau A., Tolosa E. Impact of dopamine transporter SPECT using 123I- Ioflupane on diagnosis and management of patients with clinically uncertain Parkinsonian syndromes. Mov Disord, 2004, 19, 10 , 1175-1182.

7. Coelho M., Ferreira J., Sampaio C. Parkinson's Disease. In: Evidence-based neurology: Management of neurological disorders. Candelise L., Hughes R., Liberati A., Uitdehaag B., Warlow C., eds, First ed, Blackwell Publishing Ltd, 2007, 199-220.

8. Dalwi A, Bloomfield S. Parkinson-plus syndromes. eMedecine, Oct 2005.

9. Gancher S. Parkinson disease in young adults. eMedecine, Mar 2007.

10. Gosal D, Ross O, Toft M. Parkinson's disease: the genetics of a heterogeneous disorder. Eur $J$ Neurol, 2006, 13, 6, 616-27.

11. Hauser R. Pahwa R., Lyons K., McClain T. Parkinson disease. eMedecine, Mar 2007.

12. Hauser R. Lyons K., Pahwa R., Zesiewicz T., Golbe L. Parkinson's disease. Questions and answers. Fourth ed., Merit Publish. Int, 2003, 9-47.

13. Jankovic J., Rajput A., McDermott M., Perl D. The evolution of diagnosis in early Parkinson's disease. Arch Neurol, 2000, 57, 3, 369-372.

14. Muller T., Kuhn W., Przuntek H. Non-motor symptoms of Parkinson disease. Significant impact on quality of life- using possible treatments. Fortschr Med, 1997, 115, 14, 45-48.

15. Olanow C., Watts R., Koller W. An algorithm (Decision tree) for the management of Parkinson's disease: treatment guidelines. Neurology, 2001, 56, 11, suppl 5, 54- 60 .

16. Simuni T. Diagnosis and management of Parkinson's disease. Medscape Neurology \& Neurosurgery, 2007, www.medscape.com. 